\title{
Study on Multi Objective Optimization Method and It Application in Aviation and Deceleration Device
}

\author{
Yu Guang-bin ${ }^{1 *}$, Song Ye ${ }^{1}$, Duan Yuan-wang ${ }^{1}$, Zhao Xing-fu ${ }^{2}$, Qu Zhigang ${ }^{1}$ and \\ Sun Yong-guo ${ }^{1}$ \\ ${ }^{1}$ School of Mechanical Engineering,Harbin University of Science and Technology, \\ Heilongjiang, P.R. China, 150080 \\ ${ }^{2}$ AVIC Harbin Dongan Engine(group) Corporation LTD., Heilongjiang, P.R. \\ China, 150066 \\ *593542635@qq.com
}

\begin{abstract}
Multi objective optimization problems in aviation with micro and small speed reducer are often quite challenging because these problems often involve multiple and conflicting objectives. To solve the multi-objective optimization problems (MDOP), in this paper, we propose a new multi-objective differential evolution (MODE) variant, named improved double population differential cultural particle swarm optimization algorithm, inspired by improved strategy based on algorithm fusion. From the characteristics of cultural algorithm and particle swarm optimization (pso) algorithm, considering the advantages and disadvantages of both algorithms have strong complementarily. In the process of the evolution of the belief space, the strategy of "multi - layer space, the best choice" is adopted. In the process of the evolution of the group space, using the improved double group evolution difference method. To avoid a large number of high fitness infeasible solutions are discarded and the results of the algorithm are not ideal, and the convergence speed of the algorithm is improved. Multi objective optimization design of micro small speed reduction device for Aviation Based on CPSA.By comparison with the original design, the characteristics of the new design scheme are greatly improved.It is suggested that this paper proposed the method of multi objective optimization design based on the improved double population differential and multi layer culture particle swarm optimization is feasible.
\end{abstract}

Keywords: Micro reducer, Multi-object optimization, Double groups difference, Cultural PSO algorithm

\section{Introduction}

In modern society, the application of modern society, often encountered need to make multiple objectives in a given area as far as possible the best decision problem. For example, in the optimization design of micro - speed reducer for aviation, not only requires small size, but also requires high transmission efficiency, and to reduce the impact of the transmission of the side gap. In addition may also need to consider the reliability and maintainability, etc..However, the multi-objective optimization problem is usually restricted to each other, and the optimization of the target will result in the decrease of the effect of other targets. Therefore, it is difficult to evaluate the advantages and disadvantages of the multi-objective problem. For example, small volume is difficult to meet the high transmission efficiency, good maintainability and reliability will decrease.Therefore, in practical application, we must achieve a balance between multiple

*Corresponding Author 
expectations, and thus obtain a relatively optimized result. The optimization problem in a given region is a Multi-objective Optimization Problem(MOP).Obviously, the multi-objective optimization problem is more close to the engineering practice, but also more complex. The optimization problem in many engineering practices can be transformed into a multi-objective optimization problem. Therefore, the research on the multi-objective optimization problem is more valuable for practical application.

\section{The Improved Cultural Particle Swarm Optimization Algorithm Based on Double Population}

\subsection{Basic Ideas of Cultural Particle Swarm Algorithm}

In comparison with the algorithm of the control culture and particle swarm optimization, the particle swarm algorithm can be considered as a simplified form of the cultural algorithm. The dual population differential culture particle swarm optimization algorithm for multi-objective constrained optimization problem is shown in Figure 2-1. The particle swarm algorithm is integrated into the framework of cultural algorithm to solve the optimization problem. That is, swarm intelligence is used in the evolution of group space, considering that there are a total of $(\mathrm{g})$ objective functions, which include $\mathrm{g}$ sub populations in the population space, and the target function $f_{i}(x)(i=1,2, \cdots, q)$ as a single objective function. At the end of each iteration, each of the sub populations outputs $20 \%$ of the elite particles to the belief space. The elite particles from different sub populations constitute the belief space, and they are further adopted to generate the Pareto optimal solution.

In general, the Parcto optimal solution is generated by the last generation of the algorithm. In order to maintain the optimal solution in the process of evolution, we have designed a Parcto solution pool, which is used to store the current optimal solution, which is used to solve the optimal solution in the pool with the Pareto solution. This operation has two effects:

(1) We can keep the current Pareto solutions focus on the individual's most superior, use the best experience to guide the evolution, can improve the performance of the algorithm, accelerate the convergence.

(2) To maintain the diversity of population, this is because the individual in the pool of Parcto solution can be obtained in every generation of evolution, which can contain the optimal solution of each generation in the process of the algorithm.

Independently of each sub population in space of evolutionary operation, no information exchange relationship between the sub populations, but they evolved at the same time, must accept the guidance from the experience of belief space.

\subsection{Crossover and Niche Competition Mechanism}

2.2.1. Improved CPSA Algorithm: In order to maintain the diversity of the elite particles in the belief space, we use the cross operation to enhance the information exchange and sharing among the particles, so as to further improve the performance of the algorithm, the particle evolution crossover operation strategy is carried out according to the following formula:

$$
\begin{gathered}
X_{i}^{\prime}=\alpha \cdot X_{i}+(1-\alpha) \cdot X_{j} \\
X_{j}^{\prime}=\alpha \cdot X_{j}+(1-\alpha) \cdot X_{i}
\end{gathered}
$$

where,$\alpha$ is a random number between $(0,1) . X_{i}$ and $X_{j}$ are two parent particles, While $X_{i}^{\prime}$ and $X_{j}^{\prime}$ are the two generation of cross operation. The newly generated $X_{i}^{\prime}$ 
and $X_{j}^{\prime}$ are first compared with the parent $X_{i}$ and $X_{j}$, If $X_{i}^{\prime}$ and $X_{j}^{\prime}$ are non dominated solutions, they are replaced by two of their father,Otherwise, and will be abandoned and continue to retain their father.

2.2.2. Niche Pareto Competition Mechanism: In the multi-objective evolutionary algorithm, the optimal solution set for some problems may be very large, and it may also contain a lot of solutions, which is sometimes more difficult for all these solutions to be included in the Pareto. Therefore, it is necessary to keep the size of the non dominated set in a reasonable limit. In the evolutionary algorithm, to solve the simulation of natural biological "birds of a feather flock together" phenomenon at the same time must remain problems for the diversity of population evolution, niche technology is proposed and used widely at present is niching technology based on sharing mechanism, in this mechanism defines a shared function, which represents the degree of similarity between two individuals, two individuals are more similar, the sharing function value is greater, and vice versa smaller.

After the execution of the cross operation, a selection mechanism based on the niche Pareto competition will be performed to select the best particles for replication. The detailed selection steps are as follows: random selection of two candidate particles, as well as a number of particles containing a number of particles as a comparison. There are two possible cases and the corresponding strategies to compare each candidate particle and the comparison set:

(1) if one of the candidates is compared to the set, and the other is not, then the non dominated solution will be selected for the copy operation.

(2) if two is set to dominate or control set, then a smaller niche count the particles will be selected for replication, the niche count can by calculating the entire population of the sharing function of the sum to obtain:

$m_{i}=\sum_{i=1}^{\text {swamm }} \sum^{- \text {size }} \operatorname{sh}\left(d_{i, j}\right)$

where, $\operatorname{sh}(\cdot)$ is the sharing function, usually in the form of power function is as following:

$\operatorname{sh}\left(d_{i, j}\right)=\left\{\begin{array}{cl}1-\left(\frac{d_{i, j}}{\sigma_{\text {share }}}\right)^{a} & \text { if } d_{i, j}<\sigma_{\text {share }} \\ 0 & \text { otherwise }\end{array}\right.$

where, $\sigma_{\text {share }}$ is a niche radius, and $a$ is a constant, $d_{i, j}$ is the distance between particles $i$ and $j$ in the decision space.

above-mentioned Niche Pareto competition method can ensure the final calculated solution set can converge to the Pareto front, to avoid the premature phenomenon.

As mentioned before, the belief space to accept the elite particles, its purpose is to enable the entire group of space to share the elite particles contained in the excellent information, so in the preceding cross and the choice of operation, the belief space will guide the evolution of the group space, The implementation is achieved by adding a correction term to the speed of the group space iteration formula. The formula for the particle swarm velocity update formula is changed into the following form:

$$
v_{i, j}^{(k+1)}=v_{i, j}^{(k)}+c_{1} r_{1}\left(\text { pbest }{ }_{i, j}^{(k)}-x_{i, j}^{(k)}\right)+c_{2} r_{2}\left(\text { gbest }{ }_{i, j}^{(k)}-x_{i, j}^{(k)}\right)+c_{3} r_{3}\left(\text { gbest }_{i, j}^{(k)}-x_{i, j}^{(k)}\right)
$$

where, pbest $\quad$ is the best place for the particle $i$ to pass.(Personal experience), gbest 
represents the best position of all particles in the group (group experience): $c_{1} 、 c_{2}$ are normal, $r_{1} 、 r_{2}$ are the random number of the interval of the [0-1], while $c_{3}$ is acceleration constant term . $r_{3}$ is a random number from the interval $[0,0.5]$. gbest ${ }_{j}$ is one of the best positions of the elite particles in the group space, which is the use of competitive mechanism to choose from the belief space.

As mentioned above, in order to maintain the optimal solution of Pareto in the process of evolution, this paper uses the Pareto to add a special pool in the process of the Pareto solution. Pareto solution pool method. The program specifically assigned a piece called Pareto solution pool independent storage area In each iteration, in order to maintain the optimal solution in the solution, the Pareto solution is updated by removing all of the dominant solutions and accepting new Pareto solutions from the belief space.

CPSA algorithm is a kind of double evolution mechanism, population space and belief space in each sub population of the elite population evolution can be synchronized, the basic process is as follows:

Step1: Each individual particle swarm and related parameters initial population of space, multi-faith space initialization;

Step2: Evaluation of individual fitness value, in line with the requirements to stop, otherwise continue;

Step3:The elite population and related parameters of the initial belief space;

Step4: From the group space, $30 \%$ of the best particles are selected to update the belief space, The current belief space updates,Evaluation of multi layered belief space,Choose the best faith space,Update Pareto solution pool, update numerical $\varepsilon$, The application of niche technology to compete on the solution set, update Pareto solution pool again.The belief space output the global best solution gbest to the subgroup of space group.

Step5: Apply Influence(pop(t)) update group space,The population space is evolved by the dual population differential culture evolution algorithm, and the fitness value of individual $i$ in the population of the population is calculated,To update the velocity of individual $i$ within the population space, and to update the position of individual $i$ in the population of each particle swarm,Update the current best position of each individual in the group of particles in the pbest, and update the current best group location of each particle swarm in the group space gbest;

Step6: Evaluate the fitness value of the individual, in line with the requirements of the end, otherwise continue to perform and turn to Step 3.

\section{The Verification of Multi Objective Optimization Design Based on CPSA Algorithm in Aviation with Micro and Small Speed Reducer}

\subsection{Design Parameters of RV Reduction Device in Aviation for Variable Thickness Gear with Micro Adjustable Clearance}

The output torque of the variable thickness gear RV reduction device in aviation is 150 $\mathrm{Nm}$, Rated speed $5500 \mathrm{r} / \mathrm{min}$, Total transmission ratio 110, Gear accuracy rating of 6 . The first stage large bevel gear and pinion wheel material for 20CrMnTi, and carburizing and quenching,Tooth surface hardness is HRC60, Contact fatigue limit stress of tooth surface, The tooth contact fatigue limit stress is $\sigma_{\mathrm{H} \lim 1}=1450 \mathrm{~N} / \mathrm{mm}^{2}$. The gear tooth root bending fatigue limit stress $\sigma_{\mathrm{F} \text { lim } 1}=400 \mathrm{~N} / \mathrm{mm}^{2}$. The second stage variable thickness internal gear and external gear material is $40 \mathrm{Cr}$.and quenching and tempering treatment. 


\subsection{Design Variables}

The design parameters of the original design as the initial design variables to optimize the parameters.Design is as follows:

The influence of the parameters of the RV reduction device of the gear reducer with the micro and small sized adjustable gap: the big bevel gear tooth number is $z_{1}$, the big cone gear modulus is $m_{1}$, Big bevel gear width is $B_{1}$, Mid point helix angle is $\beta$, The small bevel gear teeth is $z_{2}$, The small gear tooth width is $B_{2}$,Variable thickness outer gear tooth number is $z_{p}$, Variable thickness outer gear modulus is $m_{2}$, Variable thickness outer gear tooth width is $B_{p}$,Variable thickness inner gear wallthickness is $B$, Meshing angle is $\alpha^{\prime}$,Modification coefficient are $y_{1}, y_{2}$, respectively. The other six design variables are the parameters of the double eccentric shaft, respectively, $d_{p 1}, d_{p 2}, d_{p 3}, l_{p 1}, l_{p 2}, l_{p 3}$, Their meaning is shown in Figure 3-1.

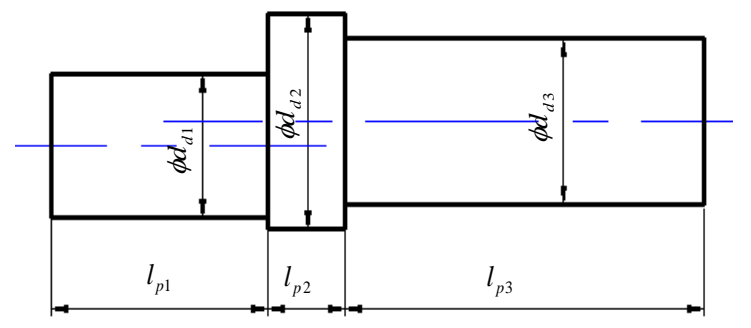

Figure 3-1. The Design Variables of Off-Center Shaft

In dealing with design variables, since aviation within micro adjustable gap thickening gear meshing RV reduction device structure is very complicated, it can only do a certain simplification, which does not affect the validity of the calculation results. So the design variables as follows:

$$
\begin{aligned}
& x=\left[x_{1}, x_{2}, x_{3}, \ldots \ldots, x_{19}\right]^{T} \\
= & {\left[z_{1}, m_{1}, B_{1}, \beta, z_{2}, B_{2}, z_{p}, m_{2}, B_{p}, B, \alpha^{\prime}, y_{1}, y_{2}, d_{p 1}, d_{p 2}, d_{p 3}, l_{p 1}, l_{p 2}, l_{p 3}\right]^{T} }
\end{aligned}
$$

Table 3-1. The Initial Parameters and Optimum Results of Optimum Design of Special Reducer

\begin{tabular}{c|c|c|c|c|c|c|c|c|c|c}
\hline $\begin{array}{c}\text { Design } \\
\text { variable }\end{array}$ & $x_{1}$ & $x_{2}$ & $x_{3}$ & $x_{4}$ & $x_{5}$ & $x_{6}$ & $x_{7}$ & $x_{8}$ & $x_{9}$ & $x_{10}$ \\
\hline $\begin{array}{c}\text { initial } \\
\text { parameters }\end{array}$ & 90 & 1 & 10 & 35 & 34 & 10 & 44 & 1.14 & 16 & 8 \\
\hline $\begin{array}{c}\text { Design } \\
\text { variable }\end{array}$ & $x_{11}$ & $x_{12}$ & $x_{13}$ & $x_{14}$ & $x_{15}$ & $x_{16}$ & $x_{17}$ & $x_{18}$ & $x_{19}$ & \\
\hline $\begin{array}{c}\text { initial } \\
\text { parameters }\end{array}$ & 52.5 & 1.14 & 0.57 & 6.5 & 10 & 7 & 11 & 6 & 14 & \\
\hline
\end{tabular}

\subsection{Determine of the Objective Function}


Since the reduction gear transmission is used for aviation equipment, requiring small size, light weight, small size,so regard small size as one of the optimization target; In the design of the planetary transmission mechanism with less teeth difference, we should first meet the condition of the tooth profile without interference,Generally use positive transmission and at the same time reduce coefficient of tooth top height,But the positive transmission will make the meshing transmission angle $\alpha^{\prime}$ increase, and the increase of meshing angle will cause the bearing radial force increases, so that the bearing life is reduced, so that the transmission efficiency is reduced.In the case of the minimum transmission device as the design objective, the contradiction is more prominent. The reduction of the tooth top high coefficient will decrease the degree of coincidence of the transmission, so that the bearing capacity of the transmission can be reduced.But because there are meshing phenomenon of many teeth in the small tooth difference transmission. As long as the coincidence degree meets the basic requirements of the design and can work properly, there is no need to pursue a large degree of coincidence.So the meshing angle small become another optimization goal.In order to make the main reducer transmission torque biger, the small bevel gear should make the allowable power maximum of according to the contact strength calculate.So the contact strength of the small bevel gear as the third optimization objectives.One of the optimization objectives is to get the minimum size of the RV reduction device with the micro small size adjustable gap gear.The main parameters influencing the volume is big, the small bevel gear volume $V_{1}, V_{2}$.Variable thickness external gear volume $V_{3}$ and Variable thickness internal gear volume $V_{4}$. In order to simplify the calculation,In this paper, the volume of arc tooth bevel gear is approximate to the diameter of the middle diameter of the gear tooth,With bevel gear tooth width to calculate the height of the cylinder.Set objective function:

$$
\begin{aligned}
& \min f_{1}(x)=V_{1}+V_{2}+V_{3}+V_{4}=0.78539\left(\frac{R_{m}}{R_{c}}\right)^{2} \times\left(d_{a 1}^{2}+d_{a 2}^{2}\right) \cdot b / \cos \left(0.5 \beta_{m}\right) \\
& +m^{2}\left(\frac{i_{z} z_{d}}{i_{1}}\right)^{2} B+4 \cdot(B+1)\left(2 B_{P}+\Delta\right)\left(m \frac{i_{z} z_{d}}{i_{1}}+B+1\right)
\end{aligned}
$$

where:

$R_{m}$-Average cone distance of bevel gears, $R_{m}=R_{e}-b / 2 ;$

$R_{e} \longrightarrow$ outer cone distance of Bevel gears, $R_{e}=0.5 m_{t} z_{1} \sqrt{1+i}$;

$i-$ Bevel gear transmission ratio;

$d_{a 1} \longrightarrow$ The small bevel gear big end

diameter: $d_{a 1}=m_{t}\left[z_{1}+2 \cos (\operatorname{arc} \cot i)\right]$;

$d_{a 2}-$ The big bevel gear big end diameter: $d_{a 2}=m_{t}\left[i z_{1}+2 \cos (\arctan i)\right]$;

$\Delta-$ Structure constant;

$i_{z} \longrightarrow$ Total transmission ratio.

The second optimization goal is to get the smallest meshing Angle,The objective function $f_{2}(x)$ is set up by no side gap mesh equation.

$\min f_{2}(x)=\operatorname{inv} \alpha^{\prime}=\operatorname{inv} \alpha+2 \frac{\left(y_{2}-y_{1}\right) \tan \alpha}{z_{d}}$ 
where:

$$
\begin{aligned}
& \alpha-\text { Dividing circle pressure Angle; } \\
& z_{d}-\text { Tooth difference. }
\end{aligned}
$$

where, inv $\alpha^{\prime}=\tan \alpha^{\prime}-\alpha^{\prime}$, After calculating the inv $\alpha^{\prime}$, the numerical approximation method is used to obtain the $\alpha^{\prime}$. The third optimization goal is to get the maximum bevel gear contact strength, Therefore, the target function $f_{3}(x)$ can be set up:

$$
\left.\begin{array}{l}
\max f(x)=\frac{m}{1.91 \times 10^{5}} \times \frac{\sigma_{H P}^{2} d_{v m 1} d_{m 1} b}{\left(Z_{H} Z_{B} Z_{\varepsilon \beta} Z_{K}\right)^{2}\left(K_{A} K_{V} K_{H \beta} K_{H \alpha}\right)} \\
\sigma_{H P}=\frac{\sigma_{H \lim }}{S_{H \min }} Z_{N} Z_{L V R} Z_{X} Z_{W}
\end{array}\right\}
$$

where:

$$
\begin{aligned}
& n_{1} \longrightarrow \text { The small bevel gear speed; } \\
& d_{V m 1} \longrightarrow \text { Small spiral bevel gear tooth width midpoint of } \\
& \text { equivalent standard pitch circle diameter of gears; } \\
& \sigma_{H P} \longrightarrow \text { Small bevel gear allowable contact stress; } \\
& d_{m 1} \longrightarrow \text { Small spiral bevel gear tooth width midpoint of dividing } \\
& \text { circle diameter; } \\
& Z_{H}-\text { Coefficient of node area; } \\
& Z_{E} \longrightarrow \text { Material elasticity coefficient; } \\
& Z_{K} \longrightarrow \text { Bevel gear coefficient; } \\
& Z_{\varepsilon \beta} \quad \text { Coincidence of the contact strength calculation andhelix } \\
& \text { angle coefficient; } \\
& K_{A} \longrightarrow \text { Usage coefficient; } \\
& K_{V} \text { Dynamic load coefficient; } \\
& K_{H \alpha}-\text { Life coefficient of contact strength calculation; } \\
& K_{H \beta} \longrightarrow \text { Dimension coefficient of contact strength calculation; } \\
& Z_{L V R}-\text { Lubricating oil film influence coefficient; } \\
& Z_{W} \longrightarrow \text { Work hardening coefficient; } \\
& S_{H \text { m in }} \text { The minimum safety coefficient of contact strength. }
\end{aligned}
$$

\subsection{The Establishment of the Constraint Condition}

Not all combinations in the design are feasible, practical solution must satisfy the conditions stipulated in the design specifications and standards, and other conditions. 
These conditions into two main categories: geometric constraints, such as size, shape constraints, etc.; Performance constraints, such as stress constraints. Some constraint conditions are as follows:

(1) geometric constraints and coincidence degree constraints:

The degree of coincidence of a gear transmission gear with a bevel gear is as follows: the longitudinal overlap degree is more than 1.25.namely

$g_{1}(x)=R_{e} b \tan \beta_{m} /\left(R_{m} \pi m_{t}\right) \geq 1.25$

Coincidence degree constraint conditions of the secondary variable thickness gear is:

$g_{2}(x)=1.1-\frac{1}{2 \pi}\left(\left(z_{c}-z_{p}\right) \tan \alpha_{t}^{\prime}+z_{p} \tan \alpha_{a t p 2}-z_{c} \tan \alpha_{\text {atc } 0}\right)-b_{c} \sin \beta /\left(\pi m_{t}\right)>0$

where:

$z_{c} \longrightarrow$ Tooth number of variable thickness internal gear;

$z_{p} \longrightarrow$ Tooth number of variable thickness external gear;

$b_{c}$ - Tooth width of variable thickness internal gear $(\mathrm{mm})$;

$\beta-$ Helix angle of variable thickness gear $\left({ }^{\circ}\right)$;

$\alpha_{a t \beta}-$ The big end section top gear pressure angle of variable

thickness external gear $\left(^{\circ}\right)$;

$\alpha_{a t \theta}-$ The small end section tooth top pressure angle of

variable thickness internal gear $\left({ }^{\circ}\right)$.

Among them, corner mark $p$ represents the variable thickness external gear, $c$ represents the variable thickness internal gear,2 represents the big end section of variable thickness gear, 0 represents the small end section of variable thickness gear .Its value can be calculated by formula following:

$$
\alpha_{a t p 2}=\arccos \frac{m_{t} z_{p} \cos \alpha_{t p}}{2 r_{a p 2}} \quad \alpha_{a t c 0}=\arccos \frac{m_{t} z_{c} \cos \alpha_{t c}}{2 r_{a c 0}}
$$

(2) tooth top thickness constraint:

$g_{3}(x)=0.4 m_{12}-s_{a 1}>0$

where: $s_{a 1} \longrightarrow$ The big bevel gear tooth top thickness $(\mathrm{mm})$.

$g_{4}(x)=0.4 m_{12}-s_{a 2}>0$

where: $s_{a 2}-$ small bevel gear tooth top thickness $(\mathrm{mm})$.

Secondary internal meshing thickening gear tooth top thick constraints:

Due to thicken the big end cross section of external gear and internal gear modification coefficient is big,So the big end of tooth top thickness thinner than the other cross section. Just consider the big end of cross section thickness of the establishment of tooth top constraints.

Thickening of external gear and internal gear addendum thickness constraints are:

$$
\begin{aligned}
& g_{5}(x)=0.4 m_{t}-\frac{\cos \alpha_{t p}}{\cos \alpha_{a t p 2}} m_{t}\left(\frac{\pi}{2}+2 x_{t p 2} \tan \alpha_{t} \cos \sigma-z_{p}\left(i n v \alpha_{a t p 2}-i n v \alpha_{t p}\right)\right)>0 \\
& g_{6}(x)=0.4 m_{t}-\frac{\cos \alpha_{t c}}{\cos \alpha_{a t c 2}} m_{t}\left(\frac{\pi}{2}-2 x_{t c 2} \tan \alpha_{t} \cos \sigma+z_{c}\left(i n v \alpha_{a t c 2}-i n v \alpha_{t c}\right)\right)>0
\end{aligned}
$$


(3) internal meshing thickening gear tooth profile overlapping interference constraint:

Thickened transmission device in the internal meshing gear pair is a tooth difference transmission, it is easy to appear tooth profile overlapping interference phenomenon.Due to the thickening gear each section along the axial displacement coefficient of linear change, Therefore, the establishment of tooth profile overlapping interference constraints, As long as its big and small two end section does not appear tooth profile overlap phenomenon, the cross sections will inevitably meet other requirements. Thickened internal meshing gear pair small end section and big end section of tooth profile overlapping interference does not occur constraints respectively are:

$g_{7}(x)=z_{p}\left(\delta_{p 0}+i n v \alpha_{a p 0}\right)-z_{c}\left(\delta_{c 0}+i n v \alpha_{a c 0}\right)+\left(z_{c}-z_{p}\right) i n v \alpha_{t}^{\prime}>0$

where:

$$
\begin{gathered}
\delta_{p 0}=\operatorname{arcco}\left(\frac{d_{a c 0}^{2}-d_{a p 0}^{2}-4 a^{2}}{4 a d_{a p 0}}\right) \\
\delta_{c 0}=\operatorname{arcco}\left(\frac{d_{a c 0}^{2}-d_{a p 0}^{2}+4 a^{2}}{4 a d_{a c 0}}\right) \\
g_{8}(x)=z_{p}\left(\delta_{p 2}+i n v \alpha_{a p 2}\right)-z_{c}\left(\delta_{c 2}+\operatorname{inv} \alpha_{a c 2}\right)+\left(z_{c}-z_{p}\right) \operatorname{inv} \alpha_{t}^{\prime}>0
\end{gathered}
$$

where, $\delta_{p 2}, \delta_{c 2}$ can be reference to the calculation formula of $\delta_{p 0}$ and $\delta_{c 0}$.

(4) Transition curve interference constraint:

The interference of the transition curve of the transition curve is mainly determined by the radius of curvature radius of the cutting tool and the limiting engagement point of the cutter, the greater the radius of curvature, the more possibility of interference. The variation of the radius of curvature of the cutting tool and the cutting edge of the cutter is changed with the variation of the coefficient of variation. The greater the coefficient of variation is, the more the base circle of the gear is to be cut off, the greater the radius of curvature is, the more easily the transition curve interference occurs. That is to say, the change of internal bevoloid gear and gear big endian prone to transition curve interference, when constraints are set up, just consider the heel section of the gear. Is the constraint condition of thick in the gears and gear end section without transition curve interference respectively:

$$
\begin{aligned}
& g_{9}(x)=\left(z_{c}-z_{0}\right) \tan \alpha_{0 c 2}^{\prime}+z_{0} \tan \alpha_{a 0}-\left(z_{c}-z_{p}\right) \tan \alpha_{t}^{\prime}-z_{p} \tan \alpha_{a p 2}>0 \\
& g_{10}(x)=z_{c} \tan \alpha_{a c 2}-\left(z_{c}-z_{p}\right) \tan \alpha_{t}^{\prime}-\left(z_{p}+z_{0}\right) \tan \alpha_{0 p 2}^{\prime}+z_{0} \tan \alpha_{a 0}>0
\end{aligned}
$$

where:

$$
\begin{aligned}
& z_{0}-\text { Cutter tooth number; } \\
& \alpha_{a 0}-\text { Cutter tooth top pressure angle }\left(^{\circ}\right) ; \\
& \alpha_{0 c 2}^{\prime}-\text { The cutting engagement angle of the cutting of thick inner gear }
\end{aligned}
$$

$$
\alpha_{0 p 2}^{\prime}-\text { Cutting engagement angle when cutting thick outer gear }\left({ }^{\circ}\right) \text {; }
$$

(5) Gear strength constraint

Strength constraint conditions are the most important in all of the constraints, and also the largest of the workload. In calculating the safety factor of gear strength, the calculation formula of Sun Yuanrao's formula is used to calculate the coefficient of the strength formula. This will make the optimization design of the program become complex, 
the calculation time is greatly increased, but it can guarantee the reliability of the optimization calculation, so that the optimization design scheme is practical.

There are six of the strength of the micro - and small - speed reduction device, they are respectively:

$$
\begin{aligned}
& g_{11}(x)=1.25-s_{h 12}>0 \\
& g_{12}(x)=1.25-s_{f 1}>0 \\
& g_{13}(x)=1.25-s_{f 2}>0 \\
& g_{14}(x)=1.25-s_{h c p}>0 \\
& g_{15}(x)=1.25-s_{f p}>0 \\
& g_{16}(x)=1.25-s_{f c}>0
\end{aligned}
$$

where:

$s_{h 12}-$ There are six of the strength of the micro and small speed reduction device, they are respectively;

$s_{f 1} 、 s_{f 2} \longrightarrow$ Respectively, the safety factor, the small bevel gear root bending fatigue strength;

$s_{h c p}$ The safety factor of the tooth surface contact fatigue strength of the two

$$
\text { stage variable thickness gear; }
$$

$s_{f p} 、 s_{f c}$ Are the safety coefficient becomes thicker, the inner gear tooth root bending fatigue strength

(6) Boundary constraint

In order to make the optimized results have practical significance, according to the design experience of the drive device and the relevant design specification, we give a certain range of each design variable, that is, the boundary conditions are obtained:

$\underline{x}_{i} \leq x_{i} \leq \bar{x}_{i} \quad(i=1,2, \ldots, 19)$

where:

$$
\begin{aligned}
& \underline{x}_{i}-\text { Lower bound of design variables; } \\
& \bar{x}_{i}-\text { Upper bound of design variables 。 }
\end{aligned}
$$

\subsection{Optimize the Design of the Program}

Modulus and the number of teeth are discrete variables in the design variables, Calculations has been processed ,according to the continuous variable process, the optimization results are rounded for making the modulus according to the recommend series, the teeth are rounded . we adopt an improved double group difference culture particle swarm fusion algorithm. This optimization program was writen with Visual c ++ 6.0 language, its program block diagram as shown in Figure 3-2, optimization program is omitted. 


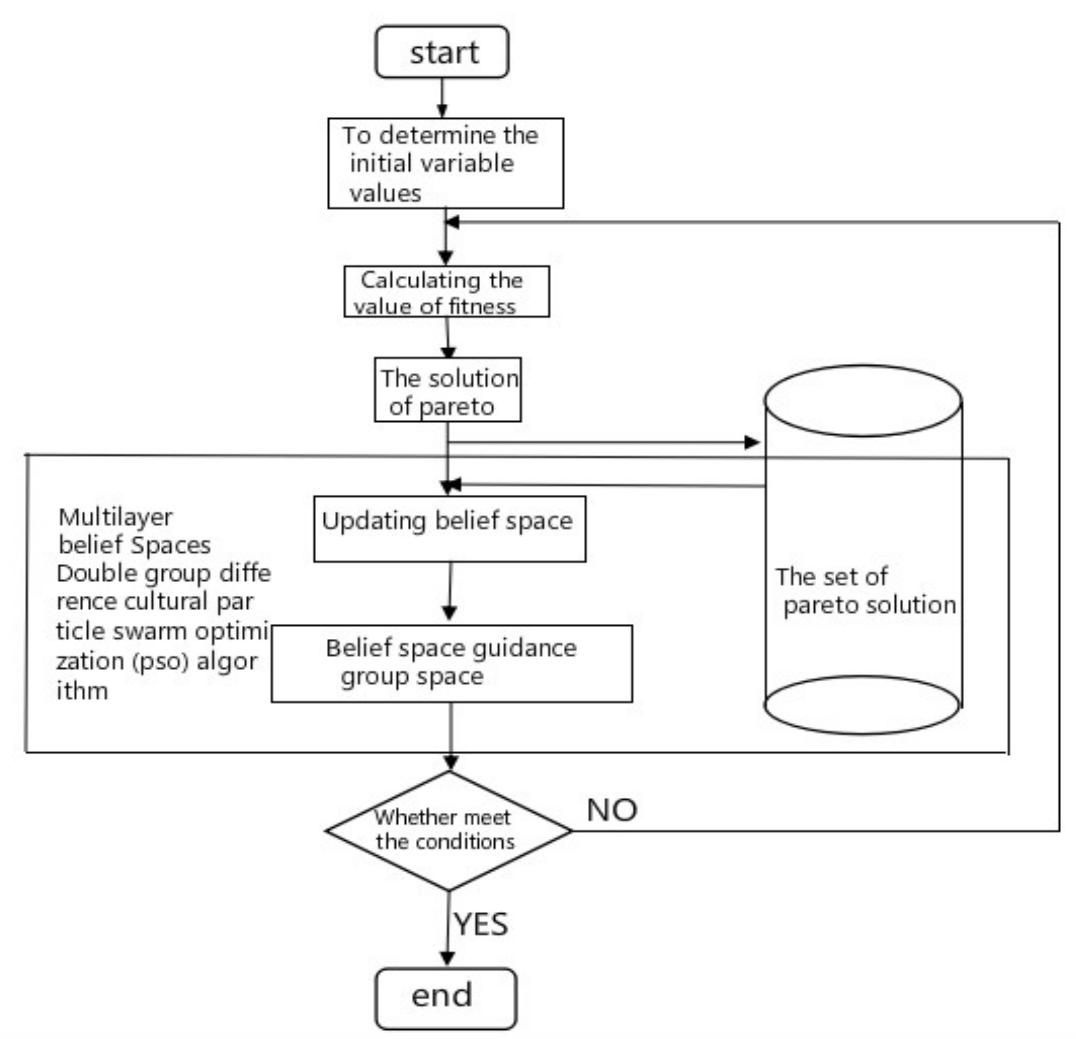

\section{Figure 3-2. The Program Diagram of Dynamic Optimum of Aviatic Micro RV Reducer}

\subsection{Contrast Optimization Results}

The design parameters of the original design scheme are optimized by the initial parameters of the design variables.The parameters of the optimized parameters are compared with that of Table 3-2.

Table 3-2. The Initial Parameters and Optimum Results of Optimum Design of Special Reducer

\begin{tabular}{c|c|c|c|c|c|c|c|c|c|c}
\hline $\begin{array}{c}\text { Design } \\
\text { variable }\end{array}$ & $x_{1}$ & $x_{2}$ & $x_{3}$ & $x_{4}$ & $x_{5}$ & $x_{6}$ & $x_{7}$ & $x_{8}$ & $x_{9}$ & $x_{10}$ \\
\hline $\begin{array}{c}\text { initial } \\
\text { parameters }\end{array}$ & 90 & 1 & 10 & 35 & 34 & 10 & 44 & 1.14 & 16 & 8 \\
\hline $\begin{array}{c}\text { optimization } \\
\text { results }\end{array}$ & 92 & 0.92 & 8.23 & 35.05 & 37 & 8.31 & 44 & 1.257 & 16 & 6.545 \\
\hline $\begin{array}{c}\text { Design } \\
\text { variable }\end{array}$ & $x_{11}$ & $x_{12}$ & $x_{13}$ & $x_{14}$ & $x_{15}$ & $x_{16}$ & $x_{17}$ & $x_{18}$ & $x_{19}$ & \\
\hline $\begin{array}{c}\text { initial } \\
\text { parameters }\end{array}$ & 52.5 & 1.14 & 0.57 & 6.5 & 10 & 7 & 11 & 6 & 14 & \\
\hline $\begin{array}{c}\text { optimization } \\
\text { results }\end{array}$ & $\begin{array}{c}54.6 \\
2\end{array}$ & 1.258 & 0.652 & 6.1 & 9.33 & 7.12 & 10.1 & 6.06 & 14.31 & \\
\hline
\end{tabular}

In order to verify whether the optimized structure scheme has better dynamic characteristics than the original design scheme,dynamic analysis of the new structure of the reduction device after the dynamic optimization,the results are listed in Table 3-3. 
Table 3-3. Performance Comparison between the Original Reducer and the Optimized One

\begin{tabular}{c|c|c|c}
\hline & $\begin{array}{c}\text { radial size } \\
(\mathrm{mm})\end{array}$ & $\begin{array}{c}\text { the biggest modal } \\
\text { flexibility } \times 10^{-6} \\
(\mathrm{rad} /(\mathrm{N} \cdot \mathrm{mm}))\end{array}$ & $\begin{array}{c}\text { the modal } \\
\text { flexibility } \\
\text { evenness }\end{array}$ \\
\hline $\begin{array}{c}\text { Before } \\
\text { optimization }\end{array}$ & 81.5 & 2.78632 & 3.5764 \\
\hline $\begin{array}{c}\text { after } \\
\text { optimization }\end{array}$ & 79.5 & 2.33922 & 3.1262 \\
\hline
\end{tabular}

Table 3-3, the optimized design scheme of the reduction device is superior to the optimized design scheme.The four order modal flexibility risk from $2.78632 * 10-6 \mathrm{rad} /$ $\mathrm{N} \mathrm{mm}$ ) to reduce the optimized $2.33922 * 10-6 \mathrm{rad} /(\mathrm{N} \mathrm{mm})$, modal flexibility evenness decreased from 3.5764 to 3.1262 after optimization,to illustrate the distribution of each modal flexibility is more uniform.

\section{Conclusion}

In this paper, a double group difference multi - layer culture particle swarm optimization algorithm is proposed, for the characteristics of large number of parameters and the mutual restriction of the large number of variable thickness gear RV reduction device for aviation.In the process of the evolution of the belief space, the strategy of "multi - layer space, the best choice" is adopted,In the process of the evolution of the group space, using the improved double group evolution difference method.To avoid a large number of high fitness infeasible solutions are discarded and the results of the algorithm are not ideal, and the convergence speed of the algorithm is improved.

Multi objective optimization design of micro small speed reduction device for Aviation Based on CPSA. The optimization program was compiled with $\mathrm{VC}++6$, multi objective optimization design for the RV reduction device of the micro small sized adjustable gap variable - thickness gear reducer is carried out.By comparison with the original design, the characteristics of the new design scheme are greatly improved,It is suggested that this paper proposed the method of multi objective optimization design based on the improved double population differential and multi layer culture particle swarm optimization is feasible.

\section{Acknowledgments}

This work supported by the Key Program of National Natural Science Foundation of Heilongjiang No.ZD201309, and Supported by Heilongjiang Natural Science Funds for Distinguished Young Scholar (Grant No. JC2015013 ; JC2014020 ), and Supported by Harbin Natural Science Funds for Distinguished Young Scholar (Grant No. 2015RAYXJ001), and Project supported by the Maor International Joint Research Program of China (Grant No. 2013DFA71120). Professor Yu Guang-bin as the corresponding author supervised this paperwork is also appreciated. 


\section{References}

[1] L. Yung-Chien, H. Kao2Shing and W. F.-Sheng, "Hybrid differential evolution with multiplier updating method for nonlinear const rained optimization problems", Proceedings of the CEC'2002. Piscataway, New Jersey, vol. 1, (2002), pp. 872-877

[2] B.-Y. Li, Y.-S. Xiao and Q.-D.Wu, "A kind of hybrid algorithm based on particle swarm optimizationalgorithm for constrained optimization problems”, Control and decision making, vol.19, no. 7, (2004), pp.804-807

[3] Meng Hong-Yun, Zhang Xiao-Hua and Liu San-Yang, "Double population differential evolution algorithm for constrained multi-objective optimization problems", Chinese Journal of Computers, vol. 31, no. 2, (2008), pp. 228-235.

[4] Gao Fang, "Study on intelligent particle swarm optimization algorithm", Doctoral dissertation of HarbinInstitute of Technology, (2008).

[5] Q. He and L.Wang, "A Hybrid Particle Swarm Optimization with a Feasibility-based Rule for Constrained Optimization",Applied Mathematics and Computation, vol. 186, no. 2, (2007), pp.1407-1422.

[6] Z. Eckart, K.A. Deb, "Comparison of multi-objective evolutionary algorithms:empirical results", [J]. evolutionary computation, vol. 8, no. 2, (2000), pp. 173-195. 
International Journal of Smart Home

Vol. 10, No. 5 (2016) 